\title{
RESEARCH
}

Open Access

\section{Evaluation of the novel liver micronucleus assay using formalin-fixed tissues}

Shuichi Hamada ${ }^{* *}$ (D), Miyuki Shigano ${ }^{1}$, Satoru Kawakami ${ }^{2}$, Maya Ueda ${ }^{3}$, Hajime Sui $^{4}, K^{2}$ Katsuya Yamada ${ }^{5}$, Soichiro Hagio ${ }^{6}$, Ayaka Momonami ${ }^{7}$, Akihisa Maeda ${ }^{8}$, Yukari Terashima ${ }^{9}$, Wakako Ohyama ${ }^{10}$, Takeshi Morita ${ }^{11}$ and Makoto Hayashi ${ }^{12}$

\begin{abstract}
Background: The repeated-dose liver micronucleus (RDLMN) assay is an effective and important in vivo test for detecting genotoxic compounds, particularly for those that require metabolic activation to show genotoxicity. In a collaborative study by the Collaborative Study Group for the Micronucleus Test (CSGMT)/The Japanese Environmental Mutagen Society (JEMS) - Mammalian Mutagenicity Study Group (MMS), micronucleus induction of 22 chemicals with the RDLMN assay employing the collagenase digestion method was examined and reported on. Recently, we have developed a method which enables retrospective evaluation of micronucleus induction in formalin-fixed liver tissues (the formalin-fixed method) obtained in general toxicity studies completed in the past. Using this method, we were able to easily evaluate clastogenic potential of chemicals from the formalin-fixed tissues obtained in the general toxicity studies.

In this study, to evaluate the usefulness of the formalin-fixed method, we have conducted a liver micronucleus assay using the formalin-fixed liver samples obtained from the above collaborative study (18 of 22 test chemicals) and carried out a comparison with the results obtained by the collagenase digestion method.

Results: Comparison of the collagenase digestion and formalin-fixed methods was conducted using the results of the micronucleus assays with a total of 18 test chemicals which included 12 genotoxic hepatocarcinogens (Group A), 4 genotoxic carcinogens but not liver targeted (Group B), and 2 nongenotoxic hepatocarcinogens (Group C). The formalin-fixed method obtained the similar results as the collagenase digestion method in 10 out of the 12 chemicals of Group A, and all chemicals of Group B and Group C. Although the results were statistically contradictive due to different levels of concurrent negative control, the 2 other chemicals of Group A showed comparable responses between the two methods.
\end{abstract}

Conclusion: The present study shows that the formalin-fixed method is capable of detecting liver carcinogens with sensitivity equal to or higher than that of the collagenase digestion method. We recommend use of the formalinfixed method because of its capability of enabling retrospective evaluation of micronucleus induction in the formalin-fixed liver tissues obtained in general toxicity studies completed in the past.

Keywords: Micronucleus assay, Liver, Formalin-fixed tissue, Collagenase, Hepatocyte

\footnotetext{
* Correspondence: Hamada.Shuuichi@mh.medience.co.jp

${ }^{1}$ Safety Assessment Department, Nonclinical Research Center, Drug

Development Service Segment, LSI Medience Corporation, 14-1, Sunayama,

Kamisu-shi, Ibaraki 314-0255, Japan

Full list of author information is available at the end of the article
}

(c) The Author(s). 2019 Open Access This article is distributed under the terms of the Creative Commons Attribution 4.0 International License (http://creativecommons.org/licenses/by/4.0/), which permits unrestricted use, distribution, and reproduction in any medium, provided you give appropriate credit to the original author(s) and the source, provide a link to the Creative Commons license, and indicate if changes were made. The Creative Commons Public Domain Dedication waiver (http://creativecommons.org/publicdomain/zero/1.0/) applies to the data made available in this article, unless otherwise stated. 


\section{Introduction}

Although the liver is not targeted in the routine micronucleus assay, the liver is an important tissue in general toxicology studies and also in carcinogenicity bioassays because test chemicals are metabolized and on occasion activated with toxicological significance in the liver. It is reported that genotoxic rodent hepatocarcinogens [1] that require metabolic activation $[2,3]$ and/or are not detectable in rodent routine erythrocyte micronucleus assays $[4,5]$ are detectable by the liver micronucleus assay.

The micronucleus assay using the liver, which is the main organ for drug metabolism, has been known to be important but not widely used because hepatocyte (HEP) proliferation in adult rats is slow and thus micronuclei are difficult to produce. To overcome this shortcoming, partial hepatectomy $[6-8]$, mitogen treatment $[9,10]$, and the use of juvenile rats [11-14] have been introduced to the assays. All these methods have disadvantages which include complex surgical procedures, decreased metabolic activity due to the partial hepatectomy [15], risk of drug interactions for mitogen treatment [16], and a lack of maturation for metabolic activation in juvenile animals [17]. Recently, we have developed a new method, the repeated-dose liver micronucleus (RDLMN) assay, to evaluate liver micronucleus through repeated administration of test chemicals, e.g., 14-day or 28-day repeated-dose treatments [18]. This method is expected to produce an accumulation of micronucleated hepatocytes (MNHEPs) through long-term continuous treatment, although HEP turnover is slow [18].

The advantages of liver micronucleus assay are made more obvious when it is incorporated into general toxicity studies. In a recently improved formalin-fixed method, procedures to prepare samples for liver micronucleus assays have been provided [19]. Because this method enables retrospective evaluation of micronucleus induction in the formalin fixed liver tissues obtained in general toxicity studies completed in the past, clastogenic potential of chemicals from the materials obtained in the general toxicity studies are able to be easily evaluated.

In a collaborative study by CSGMT/JEMS MMS, micronucleus induction of 22 test chemicals with the RDLMN assay using the collagenase digestion method [1] was examined and reported on. In this study, the micronucleus induction of 18 of those 22 test chemicals has been reexamined using the formalin-fixed liver samples, and the results have been compared with the former collaborative study which employed the collagenase digestion method.

\section{Materials and methods}

\section{Formalin-fixed liver tissues}

Five-year-old formalin (10\% phosphate-buffered) fixed liver tissues of a previous collaborative study by CSGMT/JEMS MMS were used to evaluate the micronucleus induction of
18 chemicals with the RDLMN assay by the collagenase digestion method [20-36]. The 18 test chemicals consisted of 12 genotoxic hepatocarcinogens (Group A), 4 genotoxic carcinogens but not liver targeted (Group B), and 2 nongenotoxic hepatocarcinogens (Group C) (Table 1).

In the previous collaborative study [20-36], male $\mathrm{Crl}: \mathrm{CD}(\mathrm{SD})$ rats purchased from Charles River Japan Inc. (Atsugi, Hino or Tsukuba, Japan) were 6 weeks old at the beginning of dosing. They were housed in an air-conditioned room with a 12-h light/dark cycle and given free access to food and drinking water. The animal experiments were approved by the Institutional Animal Care and Use Committee of each testing facility prior to conducting the experiments. The rats (5/group) were administered each chemical by oral gavage in a repeated dosing regimen for 14 or 28 consecutive days. Twenty-four hours after the last administration for each time point, rats were euthanized under thiopental anesthesia. The livers were then removed from the rats and a part of each liver was used for the liver micronucleus assay employing the collagenase digestion method as previously reported [20-36]. Residual tissues were immersed into $10 \%$ phosphate-buffered formalin, and stored for approximately 5 years. They were then provided for the present investigation.

\section{Preparation of hepatocyte suspensions}

HEP-specimens were prepared from the formalin-fixed liver tissues with a slightly modified version of the previously reported method $[16,19]$. In brief, a small portion of the fixed-liver tissue was cut into approximately 3 mm-cubes with a razor and thoroughly washed with water. Approximately ten cubes were incubated in approximately $15 \mathrm{~mL}$ of $12 \mathrm{M}$ aqueous solution of potassium hydroxide (KOH; Wako Pure Chemical Industries, Ltd., Osaka, Japan) at room temperature for $16 \mathrm{~h}$ and then washed thoroughly with water to remove the $\mathrm{KOH}$-solution. The tissue cubes were then mashed, filtered through a cell strainer (pore size: $100 \mu \mathrm{m}$ ), and suspended with water to disperse HEPs. The HEP-suspensions were centrifuged at $50 \times g$ for 5 min and washed with $10 \%$ phosphate-buffered formalin. Centrifugation and washing steps were repeated 3 times or more. The pellet of the HEPs was suspended with $10 \%$ phosphate-buffered formalin to prepare an HEP-suspension.

\section{Fluorescent dyes and reagents}

Fluorescent dye, SYBR ${ }^{\bullet}$ Gold (SYGO; 10,000× concentrate in dimethyl sulfoxide) purchased from Life Technologies, Inc. (Carlsbad, CA, USA), was used to stain the isolated HEPs from the formalin-fixed tissue. One $\mathrm{mol} / \mathrm{L}(\mathrm{M})$ of Tris-hydrochloride (Tris-HCl; pH 7.5) and $0.5 \mathrm{M}$ of ethylenediamine tetraacetic acid (EDTA; pH 8.0) purchased from Wako Pure Chemical Industries, Ltd. (Osaka, Japan) were mixed to prepare a TE buffer $(10 \mathrm{mM}$ Tris- $\mathrm{HCl}$ and 1 mM EDTA, pH 7.5-8.0). SYGO was diluted 2-fold with 
Table 1 Chemical profiles used in the collaborative study by CSGMT/JEMS MMS

\begin{tabular}{|c|c|c|c|c|}
\hline Group & Chemical & Abbreviation & CAS no. & Chemical class \\
\hline \multirow[t]{12}{*}{ Group A } & Dimethylnitrosoamine & DMN & $62-75-9$ & nitroso compound \\
\hline & N-Nitrosopyrrolidine & NPYR & $930-55-2$ & nitroso compound \\
\hline & N-Nitrosodipropylamine & NDPA & $621-64-7$ & nitroso compound \\
\hline & 2,4-Dinitrotoluene & 2,4-DNT & $121-14-2$ & aromatic nitro compound \\
\hline & Quinoline & QUN & $91-22-5$ & heterocyclic compound \\
\hline & p-Dimethylaminoazobenzene & $\mathrm{DAB}$ & $60-11-7$ & azo compound \\
\hline & 2-Nitropropane & 2-NP & $79-46-9$ & alkyl nitro compound \\
\hline & Monocrotaline & MCT & $315-22-0$ & alkaloid \\
\hline & N-Nitrosomorpholine & NMOR & $59-89-2$ & nitroso compound \\
\hline & 2-Acetylaminofluorene & 2-AAF & $53-96-3$ & aromatic amine \\
\hline & Sudan I (C.I.solvent yellow 14) & Sudan I & $842-07-9$ & azo compound \\
\hline & Thioacetamide & TAA & $62-55-5$ & thioamide \\
\hline \multirow[t]{4}{*}{ Group B } & Cyclophosphamide $\mathrm{H}_{2} \mathrm{O}$ & $\mathrm{CP}$ & $6055-19-2$ & bis compound \\
\hline & Potassium bromate & $\mathrm{KBrO}_{3}$ & $7758-01-2$ & inorganic metal compound \\
\hline & $N$-Methlyl-N'-nitro-N-nitrosoguanidine & MNNG & $70-25-7$ & nitroso compound \\
\hline & Methyl methanesulfonate & MMS & $66-27-3$ & alkyl sulfonate \\
\hline \multirow[t]{2}{*}{ Group C } & Clofibrate & CFB & $637-07-0$ & chlorophenoxy compound \\
\hline & Methapyrilene $\mathrm{HCl}$ & MP & $135-23-9$ & ethylene diamine \\
\hline
\end{tabular}

Group A: Genotoxic hepatocarcinogens, Group B: genotoxic carcinogens but not liver targeted, Group C: nongenotoxic hepatocarcinogens

the TE buffer. Just before microscopic observation, the prepared HEP-suspension was mixed and stained with the same volume of a solution containing SYGO at half the original concentration. The mixtures were dropped onto clean glass slides and spread with coverslips.

\section{Microscopic observation and statistical analysis}

Each of the slide specimens stained with SYGO was observed under a fluorescent microscope with B-excitation filter (wavelength: 420-490 nm). Two thousand parenchymal HEPs were analyzed, and the number of MNHEPs was recorded $[1,18]$. At the same time, the number of mitotic phase cells among the 2000 HEPs was also recorded to calculate the mitotic index (MI).

Differences in the incidence of MNHEPs between groups of test chemicals and vehicle controls were analyzed by the conditional binomial test reported by Kastenbaum and Bowman [37] at significance levels of 5 and 1\%. The proportions of MI between the treated and control groups were analyzed using Dunnett's test. Positive/Negative status was determined based mainly on the statistical analysis of MNHEP incidence to assess the biological relevance of MNHEPs, i.e., the historical control at the laboratory where the study was conducted, as well as the dose-response relationship. The judgment of biological relevance was made in a meeting of the organizing committee of this project.
The data obtained from the formalin-fixed method were compared to the data from the collagenase digestion method in the collaborative study by CSGMT/JEMS MMS [1].

\section{Results}

Group A chemicals (genotoxic hepatocarcinogens)

We reevaluated 12 chemicals of Group A using the formalin-fixed method. As a result, all 12 chemicals were found to be positive for liver micronucleus induction (Fig. 1). When compared to the collagenase digestion method employed in the previous collaborative study, the formalin-fixed method induced almost the same levels of micronuclei in most of the chemicals as the collagenase digestion method.

Relatively higher frequencies of MNHEPs were observed in DMN, NDPA, and 2,4-DNT in the formalinfixed method, while the same trend was observed in NPYR in the collagenase digestion method. Different results were obtained in TAA and Sudan I, i.e., positive in the formalin-fixed method but negative in the collagenase digestion method.

As for MI, MI evaluated in this study by the formalin-fixed method was 0 to $0.08 \%$ in the negative control group and 0 to $0.63 \%$ in the test chemical treated group, which was equivalent to that determined previously by the collagenase digestion method ( 0 to $0.12 \%$ in the negative control group, 0 to $0.55 \%$ in the test chemical treated group) [1]. 

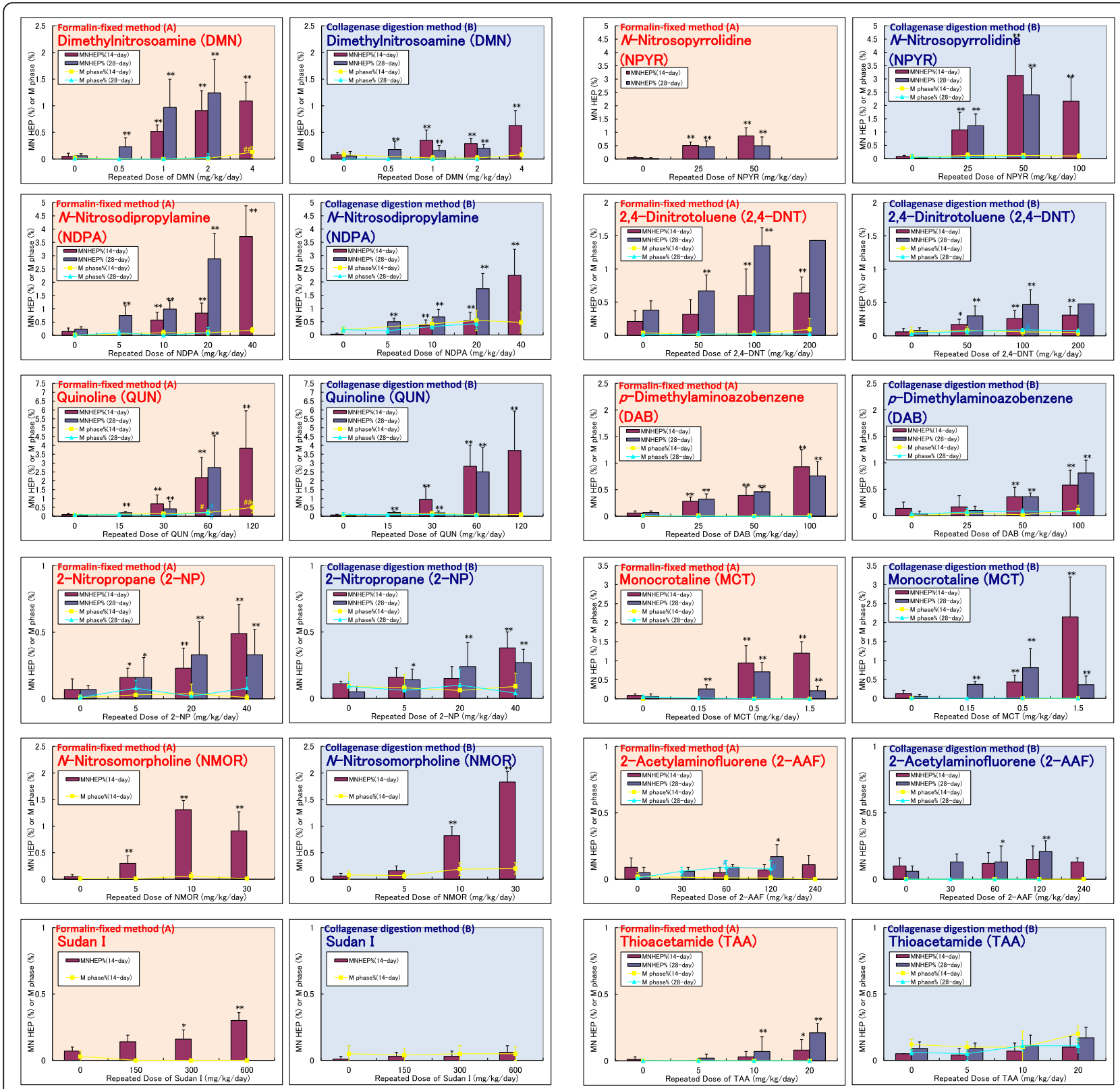

Fig. 1 Comparison of RDLMN assay results of formalin-fixed method and collagenase digestion method using Group A chemicals. Incidences of MNHEPs (\%); Comparison between formalin-fixed method (A) and collagenase digestion method reported by Hamada et al. [1] (B) in rats treated with Group A chemicals for 14 or 28 days. As for the 28-day RDLMN assay of NDPA using collagenase digestion method, it was conducted by Kissei Pharmaceutical Co., Ltd. as a collaborative study by CSGMT/JEMS MMS immediately after publication of a report by Hamada et al. [1]. Values are presented as the mean and SD. Differences in the incidences of MNHEPs between the test and vehicle control groups were analyzed by the Kastenbaum and Bowman test at significance levels of 5 and $1 \%\left({ }^{*}: P<0.05,{ }^{* *}: P<0.01\right)$. Differences in the incidences of mitotic phase cells between the test and vehicle control groups were analyzed by Dunnett's multiple comparison test at significance levels of 5 and $1 \%$ (\#: $P<0.05$, \#\#: P<0.01). Group A: genotoxic hepatocarcinogen

\section{Group B (genotoxic carcinogens but not liver targeted) and Group $\mathrm{C}$ chemicals (nongenotoxic hepatocarcinogens)}

In Group B (4 chemicals) and Group C (2 chemicals), the formalin-fixed method showed almost the same levels of micronucleus induction in all chemicals as the collagenase digestion method (Figs. 2 and 3).
As for MI, MI evaluated in this study by the formalin-fixed method was 0 to $0.06 \%$ in the negative control group and 0 to $0.06 \%$ in the test chemical treated group, which was equivalent to that determined previously by the collagenase digestion method ( 0 to $0.07 \%$ in the negative control group, 0 to $0.09 \%$ in the test chemical treated group) [1]. 

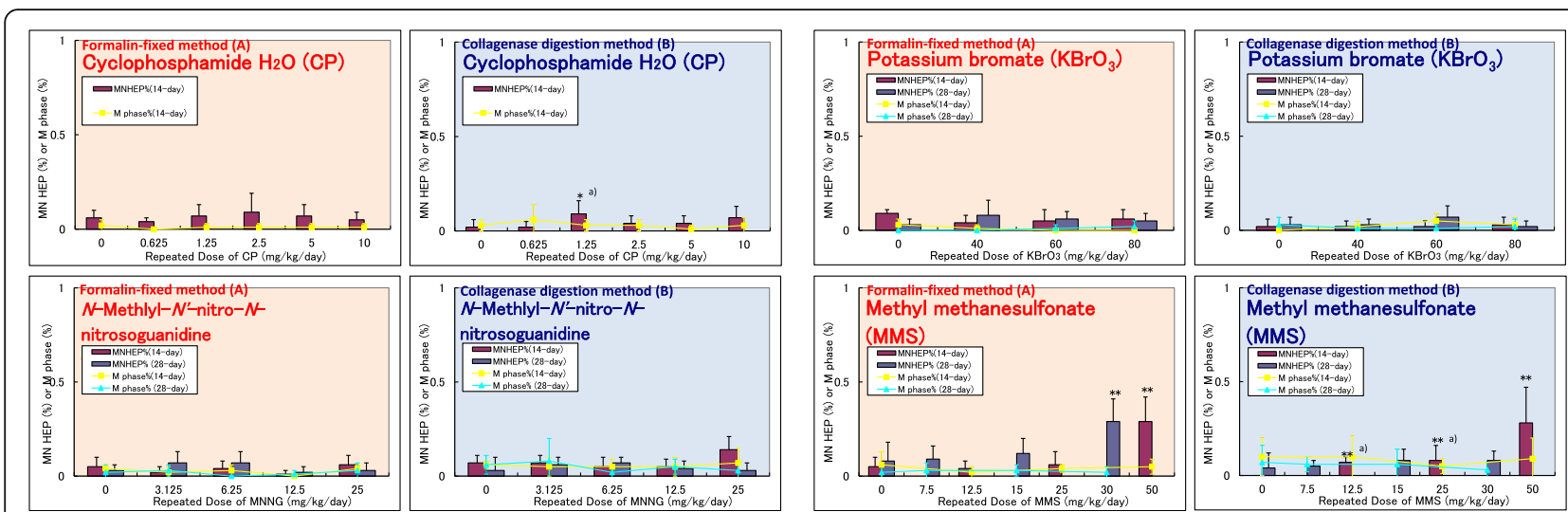

Fig. 2 Comparison of RDLMN assay results of formalin-fixed method and collagenase digestion method using Group B chemicals. Incidences of MNHEPs (\%); Comparison between formalin-fixed method (A) and collagenase digestion method reported by Hamada et al. [1] (B) in rats treated with Group B chemicals for 14 or 28 days. Values are presented as the mean and SD. Differences in the incidences of MNHEPs between the test and vehicle control groups were analyzed by the Kastenbaum and Bowman test at significance levels of 5 and $1 \%(*: P<0.05, * *$ : $P<0.01)$. Differences in the incidences of mitotic phase cells between the test and vehicle control groups were analyzed by Dunnett's multiple comparison test at significance levels of 5 and $1 \%(\#: P<0.05, \# \#: P<0.01$ ). a): Statistically significant but judged as negative because the values were within the range of the background data of negative controls in the laboratory where the MN observation was conducted. Group B: genotoxic carcinogens but not liver targeted

\section{Performance of the RDLMN assay}

The performance of the RDLMN assay is shown in Fig. 4. The sensitivity to hepatocarcinogens was determined to be $85.7 \%(12 / 14)$ by the collagenase digestion method and $100 \%(14 / 14)$ by the formalin-fixed method. Moreover, the specificity to hepatocarcinogens was $75 \%(3 / 4)$ in both methods.

\section{Discussion}

As predicted, similar results were obtained in the collagenase digestion and formalin-fixed methods in 10 out of 12 chemicals of Group A, all four chemicals of Group B, and both chemicals of Group C.

Sudan I and TAA showed negative in the collagenase digestion method while positive in the formalin-fixed method. Sudan I has been reported negative in in vitro chromosome aberration tests [38], positive in short-term bone marrow micronucleus assays [39], and positive for liver carcinogenicity
[40]. In the previous study [1, 29], Sudan I showed a tendency, though slight, toward a dose-dependent increase in liver micronucleus induction observed by the collagenase digestion method, though found negative statistically. Histopathological examination showed a remarkable HEP hypertrophy [1, 29], which indicated a possibility that collagenase treatment under such conditions may injure HEPs, leading to low sensitivity (i.e., negative result) in liver micronucleus assays. For evaluation of chemicals with strong hepatotoxicity, the formalin-fixed method which forms a single cell after formalin fixation and has lower possibility to injure HEPs as compared to the collagenase digestion method is considered more appropriate. As for TAA, the levels of micronucleus induction were comparable in the treatment groups between the two methods, but lower in the negative control of formalin-fixed samples than that of collagenase digestion ones, suggesting that the difference was due to the effect of negative control. In most of the
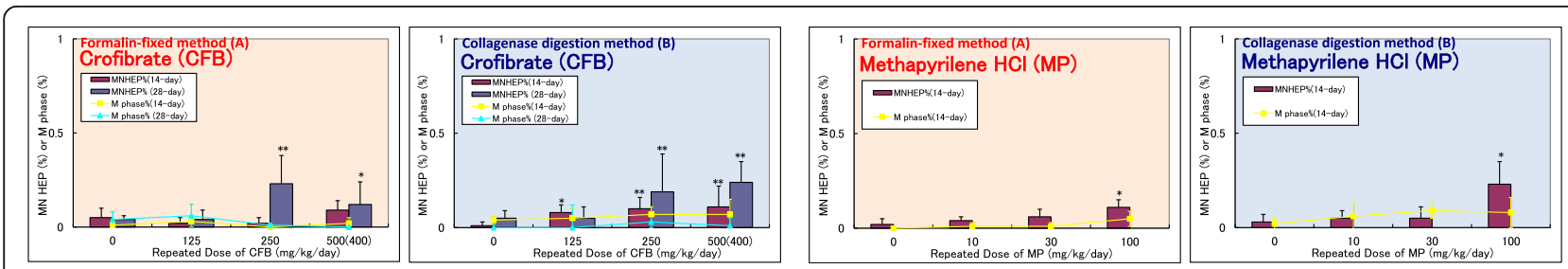

Fig. 3 Comparison of RDLMN assay results of formalin-fixed method and collagenase digestion method using Group C chemicals. Incidences of MNHEPs (\%); Comparison between formalin-fixed method (A) and collagenase digestion method reported by Hamada et al. [1] (B) in rats treated with Group C chemicals for 14 or 28 days. Values are presented as the mean and SD. Differences in the incidences of MNHEPs between the test and vehicle control groups were analyzed by the Kastenbaum and Bowman test at significance levels of 5 and $1 \%\left({ }^{*}: P<0.05,{ }^{*}: P<0.01\right)$. Differences in the incidences of mitotic phase cells between the test and vehicle control groups were analyzed by Dunnett's multiple comparison test at significance levels of 5 and $1 \%(\#: P<0.05$, \#\#: $P<0.01$ ). Group C: nongenotoxic hepatocarcinogens 


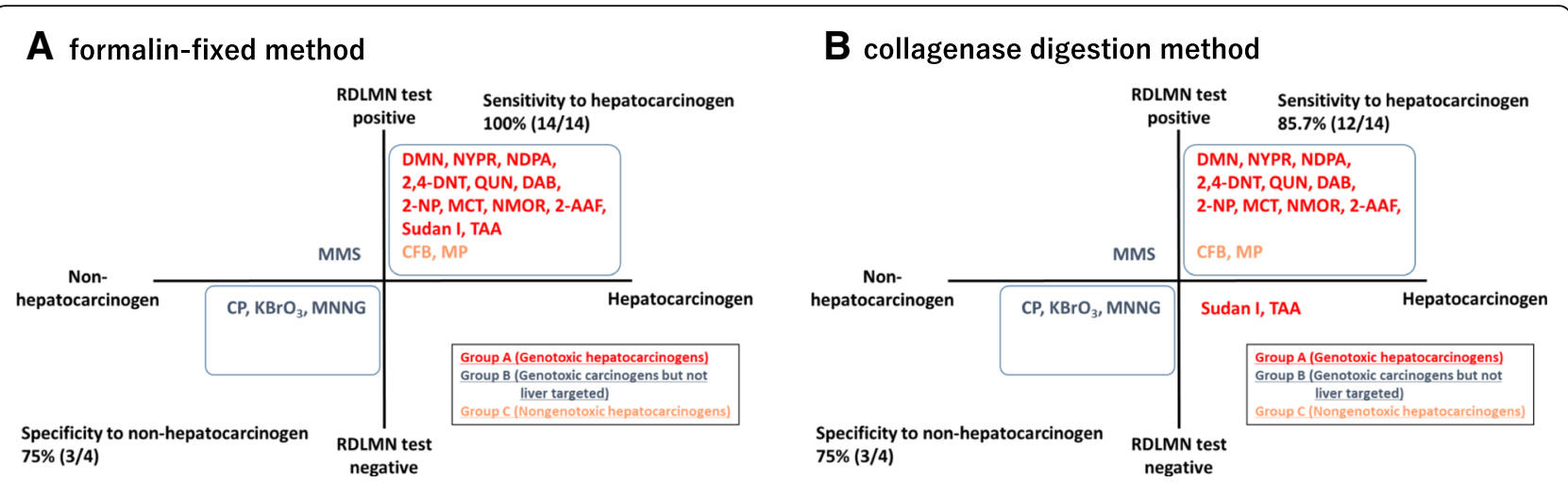

Fig. 4 Performance of the RDLMN assay: Comparison between formalin-fixed method (A) versus collagenase digestion method (B). The data of collagenase digestion method were reported by Hamada et al. [1]. Sensitivity to hepatocarcinogen $(\%)=$ (the number of chemicals that showed positive results in RDLMN assay / the number of hepatocarcinogens tested) $\times 100$. Specificity to non-hepatocarcinogen $(\%)=($ the number of chemicals that showed negative results in RDLMN assay / the number of non-hepatocarcinogens tested) $\times 100$

chemicals, micronucleus induction determined by the formalin-fixed method was equivalent to or relatively higher than that determined by the collagenase digestion method except for NPYR, for which collagenase digestion method showed a higher micronucleus induction than the formalin-fixed method. In some chemicals, same results were obtained as to the positive and negative for micronucleus induction; however, the micronucleus induction (\%) varied largely by more than 2 times between the collagenase digestion method and the formalin-fixed method. These are possibly due to the testing facilities and observers being different for each method and difference in the part of the liver where samples were collected; however, the exact cause remains unclear and further investigation is considered necessary.

As a result, sensitivity to hepatocarcinogens was slightly higher $(100 \%$ [14/14]) in the formalin-fixed method as compared to the collagenase digestion method (85.7\% [12/14]); however, the specificity to non-hepatocarcinogens did not differ between the two methods. This suggests that the formalin-fixed method has the capability of enabling detection of liver carcinogens equal to or higher than the collagenase digestion method.

MI used as an indicator of cytotoxicity was extremely low in the collagenase digestion method and formalin-fixed method, which suggested that MI is not appropriate as an indicator of cytotoxicity in the RDLMN assay. The cause of the low MI is possibly due to difference in the principals of evaluation, in which accumulation of micronucleus inducers is evaluated over the period of repeated dosing for micronucleus induction, while the number of mitotic cells over several hours before necropsy is evaluated for MI.

An integrated study that can evaluate multiple toxicity indices in the same individual animal is the ideal form of toxicity study. The formalin-fixed method has made it dramatically easier to conduct RDLMN assays by using the liver collected from the animals used in the general toxicity studies. Furthermore, it is possible to conduct a retrospective evaluation of micronucleus induction using formalin-fixed specimens of past toxicity studies. In this context, histopathological examination that is usually conducted in general toxicity studies would give direct information about cytotoxicity and HEP proliferation and indirect information about chemical exposure (more directly with toxicokinetic analysis in the case of pharmaceuticals).

Currently, evaluation by the formalin-fixed method has been commenced not only with the liver but also with the digestive tract, which is considered an effective method to facilitate sharing experimental animals between general toxicity and genotoxicity studies.

\section{Conclusion}

The present study shows that the formalin-fixed method has the capability to enable detection of micronucleus induction in HEPs equal to or higher than the collagenase digestion method. We recommend use of the formalin-fixed method not only for the above reason but also for the fact that it allows retrospective evaluation of micronucleus induction in formalin fixed liver tissues obtained in general toxicity studies completed in the past.

\footnotetext{
Abbreviations

2,4-DNT: 2,4-dinitrotoluene; 2-AAF: 2-acetylaminofluorene; 2-NP: 2nitropropane; CFB: clofibrate; CP: cyclophosphamide $\mathrm{H}_{2} \mathrm{O}$; CSGMT: the Collaborative Study Group for the Micronucleus Test; DAB: $p$ dimethylaminoazobenzene; DMN: dimethylnitrosoamine; EDTA: ethylenediamine tetraacetic acid; HEP: hepatocyte; JEMS: The Japanese Environmental Mutagen Society; $\mathrm{KBrO}_{3}$ : potassium bromate;

MCT: monocrotaline; MI: mitotic index; MMS: Mammalian Mutagenicity Study Group; MMS: methyl methanesulfonate; MNHEP: micronucleated hepatocyte; MNNG: $N$-methlyl- $N$ '-nitro- $N$-nitrosoguanidine; MP: methapyrilene $\mathrm{HCl}$; NDPA: N-nitrosodipropylamine; NMOR: N-nitrosomorpholine; NPYR: Nnitrosopyrrolidine; QUN: quinoline; RDLMN assay: repeated-dose liver
} 
micronucleus assay; SYGO: SYBR ${ }^{\oplus}$ Gold; TAA: thioacetamide; TE: $10 \mathrm{mM}$ Tris$\mathrm{HCl}$ and $1 \mathrm{mM}$ EDTA; Tris-HCl: Tris-hydrochloride

\section{Acknowledgements}

The authors thank the support by MMS/JEMS and also all participants who worked mainly on a voluntary basis. The authors are indebted to Mrs. K. Kandatsu for her critical review of the manuscript.

\section{Funding}

Not applicable.

\section{Availability of data and materials}

All data generated or analyzed during this study are included in this published article.

\section{Authors' contributions}

$\mathrm{SH}, \mathrm{MS}, \mathrm{SK}, \mathrm{MU}, \mathrm{HS}, \mathrm{KY}, \mathrm{SH}, \mathrm{AM}, \mathrm{MA}$, and $\mathrm{YT}$ performed liver micronucleus assay of compounds and they are in charge of using formalin-fixed liver samples and statistical analysis of the results obtained in the assay. SH, MS, WO, $\mathrm{TM}$, and $\mathrm{MH}$ performed comprehensive evaluation of all laboratory data. $\mathrm{SH}$ created table, fig, and manuscript. All authors have read and approved the final manuscript.

\section{Ethics approval}

The animal experiments were approved by the Institutional Animal Care and Use Committee of each testing facility prior to conducting the experiments.

\section{Consent for publication}

Not applicable

\section{Competing interests}

The authors declare that they have no competing interests.

\section{Publisher's Note}

Springer Nature remains neutral with regard to jurisdictional claims in published maps and institutional affiliations.

\begin{abstract}
Author details
${ }^{1}$ Safety Assessment Department, Nonclinical Research Center, Drug Development Service Segment, LSI Medience Corporation, 14-1, Sunayama, Kamisu-shi, Ibaraki 314-0255, Japan. ${ }^{2}$ Asahi Kasei Pharma Corporation, 632-1 Mifuku, Izunokuni-shi, Shizuoka 410-2321, Japan. ${ }^{3}$ BioSafety Research Center Inc., 582-2 Shioshinden, Iwata-shi, Shizuoka 437-1213, Japan. ${ }^{4}$ Food and Drug Safety Center, 729-5 Ochiai, Hadano-shi, Kanagawa 257-8523, Japan.

${ }^{5}$ Mitsubishi Tanabe Pharma Corporation, 2-2-50 Kawagishi, Toda-shi, Saitama 335-8505, Japan. ${ }^{6}$ Nissan Chemical Corporation, 1470 Shiraoka, Shiraoka-shi, Saitama 349-0294, Japan. ' Suntory MONOZUKURI Expert Ltd., 8-1-1 Seikadai, Seika-cho, Soraku-gun, Kyoto 619-0284, Japan. ${ }^{8}$ Toray Industries Inc., 6-10-1 Tebiro, Kamakura-shi, Kanagawa 248-8555, Japan. ${ }^{9}$ Kissei Pharmaceutical Co., Ltd., 2320-1 Maki, Hotaka, Azumino-shi, Nagano 399-8305, Japan. ${ }^{10}$ Yakult Honsha Co., Ltd., 5-11 Izumi, Kunitachi-shi, Tokyo 186-8650, Japan. " ${ }^{11}$ National Institute of Health Sciences, 3-25-26 Tonomachi, Kawasaki-shi, Kanagawa 210-9501, Japan. ${ }^{12}$ makoto international consulting, 4-23-3-1 Kamiimaizumi, Ebina-shi, Kanagawa 243-0431, Japan.
\end{abstract}

Received: 7 January 2019 Accepted: 1 April 2019

Published online: 09 May 2019

\section{References}

1. Hamada S, Ohyama W, Takashima R, Shimada K, Matsumoto K, Kawakami S, Uno F, Sui H, Shimada Y, Imamura T, Matsumura S, Sanada H, Inoue K, Muto S, Ogawa I, Hayashi A, Takayanagi T, Ogiwara Y, Maeda A, Okada E, Terashima Y, Takasawa H, Narumi K, Wako Y, Kawasako K, Sano M, Ohashi N, Morita T, Kojima H, Honma M, Hayashi M. Evaluation of the repeated-dose liver and gastrointestinal tract micronucleus assays with 22 chemicals using young adult rats: summary of the collaborative study by the collaborative study Group for the Micronucleus Test (CSGMT)/The Japanese environmental mutagen society (JEMS) - mammalian mutagenicity study group (MMS). Mutat Res. 2015;780-781:2-17.

2. Natarajan AT, Tates AD, Van Buul PP, Meijers M, NDe V. Cytogenetic effects of mutagens/carcinogens after activation in a microsomal system in vitro I. induction of chromosome aberrations and sister chromatid exchanges by diethylnitrosamine (DEN) and dimethylnitrosamine (DMN) in CHO cells in the presence of rat-liver microsomes. Mutat Res. 1976;37:83-90.

3. Ashby J, Tennant RW. Definitive relationships among chemical structure, carcinogenicity and mutagenicity for 301 chemicals tested by the U. S. NTP. Mutat Res. 1991;257:229-306.

4. Morita T, Asano N, Awogi T, Sasaki YF, Sato S, Shimada S, Sutou S, Suzuki T, Wakata A, Sofuni T, Hayashi M. Evaluation of the rodent micronucleus assay in the screening of IARC carcinogens (group 1, 2A and 2B). The summary report of the 6th collaborative study by CSGMT/JEMS.MMS. Mutat Res. 1997;389:3-122.

5. George $E$, Westmoreland C. Evaluation of the in vivo genotoxicity of the structural analogues 2,6-diaminotoluene using the rat micronucleus test and rat liver UDS assay. Carcinogenesis. 1991;12:2233-7.

6. Tates AD, Neuteboom I, Hofker M, LDen E. A micronucleus technique for detecting clastogenic effects of mutagens/carcinogens (DEN, DMN) in hepatocytes of rat liver in vivo. Mutat Res. 1980;74:11-20.

7. Tates $A D, L D e n E$. The role of short-lived lesions in the induction of micronuclei in rat liver by ethylnitrosourea and methyl methanesulfonate: the importance of experimental design. Mutat Res. 1989;210:271-9.

8. Angelosanto FA. Tissues other than bone marrow that can be used for cytogenetic analysis. Environ Mol Mutagen. 1995;25:338-43.

9. Braithwaite I, Ashby J. A non-invasive micronucleus assay in the rat liver. Mutat Res. 1988;203:23-32

10. Ashby J, Lefevre PA. The rat-liver carcinogen $\mathrm{N}$-nitrosomorpholine initiates unscheduled DNA synthesis and induces micronuclei in the rat liver in vivo. Mutat Res. 1989;225:143-7.

11. Suzuki H, Ikeda N, Kobayashi K, Terashima Y, Shimada Y, Suzuki T, Hagiwara T, Hatakeyama S, Nagaoka K, Yoshida J, Saito Y, Tanaka J, Hayashi M. Evaluation of liver and peripheral blood micronucleus assays with 9 chemicals using young rats. A study by the Collaborative Study Group for the Micronucleus Test (CSGMT)/Japanese Environmental Mutagen Society (JEMS)-Mammalian Mutagenicity Study Group (MMS). Mutat Res. 2005;583:133-45.

12. Suzuki H, Takasawa H, Kobayashi K, Terashima Y, Shimada Y, Ogawa I, Tanaka J, Imamura T, Miyazaki A, Hayashi M. Evaluation of a liver micronucleus assay with 12 chemicals using young rats (II): a study by the collaborative study Group for the Micronucleus Test/Japanese environmental mutagen society-mammalian mutagenicity study group. Mutagenesis. 2009;24:9-16.

13. Takasawa H, Suzuki H, Ogawa I, Shimada Y, Kobayashi K, Terashima Y, Matsumoto H, Aruga C, Oshida K, Ohta R, Imamura T, Miyazaki A, Kawabata M, Minowa S, Hayashi M. Evaluation of a liver micronucleus assay in young rats (III): a study using nine hepatotoxicants by the collaborative study Group for the Micronucleus Test (CSGMT)/Japanese environmental mutagen society (JEMS)-mammalian mutagenicity study group (MMS). Mutat Res. 2010;698:30-7.

14. Takasawa H, Suzuki H, Ogawa I, Shimada Y, Kobayashi K, Terashima Y, Matsumoto H, Oshida K, Ohta R, Imamura T, Miyazaki A, Kawabata M, Minowa S, Maeda A, Hayashi M. Evaluation of a liver micronucleus assay in young rats (IV): a study using a double-dosing/single-sampling method by the collaborative study Group for the Micronucleus Test (CSGMT)/Japanese environmental mutagen society (JEMS)-mammalian mutagenicity study group (MMS). Mutat Res. 2010;698:24-9.

15. Rossi AM, Romano M, Zaccaro L, Pulci R, Salmona M. DNA synthesis, mitotic index, drug-metabolising systems and cytogenetic analyses in regenerating rat liver. Mutat Res. 1987;182:75-82.

16. Parton JW, Garriott ML. An evaluation of micronucleus induction in bone marrow and in hepatocytes isolated from collagenase perfused liver or from formalin-fixed liver using four-week-old rats treated with known clastogens. Environ Mol Mutagen. 1997;29:379-85.

17. Kato R, Yamazoe Y. Sex-specific cytochrome P450 as a cause of sex and species-related differences in drug toxicity. Toxicol Lett. 1992;64/65:661-7.

18. Narumi K, Ashizawa K, Takashima R, Takasawa H, Katayama S, Tsuzuki Y, Teramoto H, Morita T, Hayashi M, Hamada S. Development of repeateddose liver micronucleus assay using adult rats: an investigation of diethylnitrosamine and 2, 4-diaminotoluene. Mutat Res. 2012;747:234-9.

19. Shigano M, Takashima R, Takasawa H, Hamada S. Optimization of specimen preparation from formalin-fixed liver tissues for liver micronucleus assays: hepatocyte staining with fluorescent dyes. Mutat Res. 2016;800:35-9.

20. Takashima R, Takasawa H, Kawasako K, Ohyama W, Okada E, Narumi K, Fujiishi Y, Wako Y, Yasunaga K, Hattori A, Kawabata M, Nakadate K, Nakagawa M, Hamada S. Evaluation of a repeated dose liver micronucleus 
assay in rats treated with two genotoxic hepatocarcinogens, dimethylnitrosamine and 2-acethylaminofluorene: the possibility of integrating micronucleus tests with multiple tissues into a repeated dose general toxicity study. Mutat Res. 2015;780-781:18-24.

21. Ogawa I, Hagio S, Furukawa S, Abe M, Kuroda Y, Hayashi S, Wako Y, Kawasako K. Evaluation of repeated dose micronucleus assays of the liver using N-nitrosopyrrolidine: a report of the collaborative study by CSGMT/ JEMS MMS. Mutat Res. 2015;780-781:25-30.

22. Terashima Y, Yokoi R, Takakura I, Saitou E, Wako Y, Kawasako K, Souma S, Tamura T. Detection of micronuclei in hepatocytes isolated from young adult rats repeatedly treated with $\mathrm{N}$-nitrosodi-n-propylamine. Mutat Res. 2015;780-781:36-40

23. Maeda A, Tsuchiyama H, Asaoka Y, Hirakata M, Miyoshi T, Oshida K, Miyamoto $Y$. Evaluation of the repeated-dose liver micronucleus assay using 2,4-dinitrotoluene: a report of a collaborative study by CSGMT/JEMS MMS. Mutat Res. 2015;780-781:41-5.

24. Uno F, Tanaka J, Ueda M, Nagai M, Fukumuro M, Natsume M, Oba M, Akahori A, Masumori S, Takami S, Wako Y, Kawasako K, Kougo Y, Ohyama W, Narumu K, Fujiishi Y, Okada E, Hayashi M. Repeated-dose liver and gastrointestinal tract micronucleus assays for quinoline in rats. Mutat Res. 2015;780-781:51-5.

25. Shimada $Y$, Sui $H$, Wako $Y$, Kawasako $K$ The Evaluation of the repeated-dose liver micronucleus assay with p-dimethylaminoazobenzene. Mutat Res. 2015;780-781:56-9.

26. Kawakami S, Araki T, Nakajima M, Kusuoka O, Uchida K, Sato N, Tanabe Y, Takahashi K, Wako Y, Kawasako K, Tsurui K. Repeated-dose liver micronucleus assay: an investigation with 2-nitropropane, a hepatocarcinogen. Mutat Res. 2015;780-781:60-3.

27. Takashima R, Takasawa H, Wako Y, Kawasako K, Yasunaga K, Hattori A, Kawabata M, Nakadate K, Nakagawa M, Hamada S. Micronucleus induction in rat liver and bone marrow by acute vs. repeat doses of the genotoxic hepatocarcinogen monocrotaline. Mutat Res. 2015;780-781:64-70.

28. Hayashi A, Kosaka M, Kimura A, Wako Y, Kawasako K, Hamada S. Evaluation of the repeated-dose liver micronucleus assay using $\mathrm{N}$-nitrosomorpholine in young adult rats: report on collaborative study by the collaborative study Group for the Micronucleus Test (CSGMT)/Japanese environmental mutagen society (JEMS) - mammalian mutagenicity study (MMS) group. Mutat Res. 2015;780-781:71-5.

29. Matsumura S, Ikeda N, Hamada S, Ohyama W, Wako Y, Kawasako K, Kasamatsu T, Nishiyama N. Repeated-dose liver and gastrointestinal tract micronucleus assays with Cl solvent yellow 14 (Sudan I) using young adult rats. Mutat Res. 2015;780-781:76-80.

30. Sui $\mathrm{H}$, Matsumoto $\mathrm{H}$, Wako $\mathrm{Y}$, Kawasako K. Evaluation of in vivo genotoxicity by thioacetamide in a 28-day repeated-dose liver micronucleus assay using male young adult rats. Mutat Res. 2015;780-781:81-4.

31. Matsumoto K, Zaizen K, Miyamoto A, Wako Y, Kawasako K, Ishida H. Evaluation of the repeated dose liver micronucleus assay using young adult rats with cyclophosphamide monohydrate: a report of a collaborative study by CSGMT/JEMS MMS. Mutat Res. 2015;780-781:90-3.

32. Okada E, Fujiishi Y, Narumi K, Kado S, Wako Y, Kawasako K, Kaneko K, Ohyama W. Evaluation of repeated dose micronucleus assays of the liver and gastrointestinal tract using potassium bromate: a report of the collaborative study by CSGMT/JEMS MMS. Mutat Res. 2015;780-781:94-9.

33. Takayanagi T, Wako Y, Kawasako K, Hori H, Fujii W, Ohyama W. Repeated dose liver and gastrointestinal tract micronucleus assays using $N$-methyl- $N^{\prime \prime}$-nitro- $N$ nitrosoguanidine in young adult rats. Mutat Res. 2015;780-781:100-6.

34. Muto S, Yamada K, Kato T, Wako Y, Kawasako K, Iwase Y, Uno Y. Assessment of methyl methanesulfonate using the repeated-dose liver micronucleus assay in young adult rats. Mutat Res. 2015;780-781:107-10.

35. Takayanagi T, Takashima R, Wako Y, Kawasako K, Tanaka Y, Hori H, Fujii W. Repeated dose liver micronucleus assay using clofibrate in young adult rats. Mutat Res. 2015;780-781:117-22.

36. Inoue K, Ochi A, Koda A, Wako Y, Kawasako K, Doi T. The 14-day repeated dose liver micronucleus test with methapyrilene hydrochloride using young adult rats. Mutat Res. 2015;780-781:123-7.

37. Kastenbaum MA, Bowman KO. Tables for determining the statistical significance of mutation frequencies. Mutat Res. 1979;9:527-49.

38. Kirkland D, Aardema M, Henderson L, Müller L. Evaluation of the ability of a battery of three in vitro genotoxicity tests to discriminate rodent carcinogens and non-carcinogens: I. sensitivity, specificity and relative predictivity. Mutat Res. 2005;584:1-256.
39. Wakata A, Miyamae Y, Sato S, Suzuki T, Morita T, Asano N, Awogi T, Kondo $\mathrm{K}$, Hayashi M. Evaluation of the rat micronucleus test with bone marrow and peripheral blood: summary of the 9th collaborative study by CSGMT/ JEMS MMS. Environ Mol Mutagen. 1998;32:84-100.

40. Gold LS. The carcinogenic potency database (CPDB). Last updated: 1 Sept 2011. http://toxnet.nlm.nih.gov/cpdb. Accessed 18 Sept 2018.

\section{Ready to submit your research? Choose BMC and benefit from:}

- fast, convenient online submission

- thorough peer review by experienced researchers in your field

- rapid publication on acceptance

- support for research data, including large and complex data types

- gold Open Access which fosters wider collaboration and increased citations

- maximum visibility for your research: over $100 \mathrm{M}$ website views per year

At BMC, research is always in progress.

Learn more biomedcentral.com/submissions 